\title{
Spectroscopic Investigation of Fluorescence Quenching Agents. Part IV: Selectivity of Nitromethane for Discriminating Between Alternant Versus Nonalternant Polycyclic Aromatic Hydrocarbons in Solvents of Differing Polarities
}

\author{
SHERYL A. TUCKER, HEATHER C. BATES, WILLIAM E. ACREE, JR., ${ }^{*}$ and \\ JOHN C. FETZER \\ Department of Chemistry, University of North Texas, Denton, Texas 76203-5068 (S.A.T., H.C.B., W.E.A.); and Cheuron Research \\ and Technology Center, Richmond, California 94802-0627 (J.C.F.)
}

\begin{abstract}
To further assess the applicability of nitromethane as a selective quenching agent for alternant vs. nonalternant polycyclic aromatic hydrocarbons in HPLC analysis, we measured the effect that it has on the fluorescence emission behavior of 96 different polycyclic aromatic hydrocarbons dissolved in a binary ethyl acetate/acetonitrile solvent mixture. Nitromethane quenching results are compared with previously reported acetonitrile, aqueous/acetonitrile, and toluene/acetonitrile solvent mixtures. Results of these measurements revealed that the "selective quenching" rule is obeyed for the vast majority of PAHs in all solvents considered thus far, with the coronene derivatives being the only major exceptions.

Index Headings: Fluorescence; Molecular structure; Spectroscopic techniques.
\end{abstract}

\section{INTRODUCTION}

Polycyclic aromatic compounds (PACs) have received considerable attention in the recent chemical literature, in part because of the widely varying carcinogenic and mutagenic properties of closely related isomers. Carcinogenic properties of select isomers, combined with an increasing awareness of environmental pollution and toxic material disposal, have prompted researchers to develop analytical methods specific for the different aromatic compounds. Current analytical methods generally employ high-resolution chromatographic techniques combined with pre-concentration via supercritical fluid ${ }^{1-5}$ or Soxhlet extraction, ${ }^{5-7}$ relatively sophisticated or lengthy isolation, or group separation schemes to obtain fractions from environmental or biological samples suitable for chromatographic analysis. Conventional chemically bonded monomeric $\mathrm{C}_{18}$ stationary phases ${ }^{8-12}$ (i.e., phases prepared with monofunctional silanes) can generally separate the various PAH molecules present according to overall size and number of aromatic rings. Monomeric $\mathrm{C}_{18}$ phases possess only limited inherent ability to separate PAH isomers on the basis of molecular shape. Enchanced shape recognition is achieved on either a "charge transfer" 13 or a polymeric $\mathrm{C}_{18}$ stationary phase ${ }^{8-12,14,15}$ (i.e., phase prepared with either difunctional or trifunctional silanes in the presence of water),

\footnotetext{
Received 18 May 1993.

* Author to whom correspondence should be sent.
}

the latter of which is believed to be molecularly ordered to some extent. Relative retention values for similar PAH structures of increasing size (i.e., naphthalene, pyrene, benzo[ghi]perylene, benzo[pqr]naphtho[8,1,2bcd]perylene, benzo[rst]dinaphtho[2,1,8,7 defg; $\left.2^{\prime}, 1^{\prime}, 8^{\prime}, 7^{\prime} \mathrm{ijkl}\right]$ pentaphene, and tetrabenzo[def,lm,qrs,yz]pyranthene) with the use of different polymeric $\mathrm{C}_{18}$ stationary and mobile phase combinations, along with the elution strengths of the eleven common HPLC solvents, are reviewed in detail elsewhere. ${ }^{15}$

Problems associated with co-eluting components in HPLC can be overcome in part by making the detector selective for each given PAC subclass. Of the four most common HPLC detectors on the market, fluorescence affords the most selectivity in that the excitation and emission wavelengths can be varied independently. While several polycyclic aromatic compounds may absorb at the same excitation wavelength, not all will emit at the wavelength(s) monitored by the detector. Utilization of selective fluorescence quenching agents further simplifies observed emission spectra by eliminating signals from undesired chemical interferences having only slightly different molecular structures. On the basis of limited fluorescence measurements for perylene, dibenzo[b,k]chrysene, dibenzo[h,rst]pentaphene, naphtho[1,2b]fluoranthene, indeno[1,2,3cd]pyrene, and 12,11-(perinaphthylene)fluoranthene dissolved in a binary aqueous/ acetonitrile mixture (20:80 percent by volume), Blümer and Zander ${ }^{16}$ noted that nitrobenzene and nitromethane selectively quenched fluorescence emission of the socalled "alternant" polycyclic aromatic hydrocarbons (PAHs). Emission intensities of the three nonalternant PAHs (e.g., naphtho[1,2b]fluoranthene, indeno[1,2,3cd]pyrene, and 12,11-(perinaphthylene)fluoranthene) were unaffected. Published studies ${ }^{17-22}$ involving over 63 PAHs have identified dibenzo[hi,wx]heptacene, benzo[k]fluoranthene, and naphtho[2,3b]fluoranthene as among the few exceptions to the so-called nitromethane selective quenching rule in the PAH6 benzenoid, fluorenoid, fluoranthenoid and "methylene-bridged" cyclopenta-PAH subclasses. More recent measurements ${ }^{23}$ revealed that nitromethane quenched fluorescence emission of all nine acenaphthylene derivatives studied thus far, which is completely contrary to what would be expected 
on the basis of the fact that the nine solutes are nonalternant PAH molecules. Despite the aforementioned exceptions, nitromethane remains a very useful, selective quenching reagent in fluorescence analysis of unknown mixtures of polycyclic aromatic compounds.

For the most part previous nitromethane quenching investigations have been confined to more polar solvents such as neat acetonitrile or binary aqueous/acetonitrile mixtures. Both systems are used routinely as mobile phases in HPLC analysis of small PAHs having six rings or less. However, larger 6-10 ring PAH molecules may require an aromatic hydrocarbon or a dichloromethane mobile phase cosolvent in order for the solutes to elute from the chromatographic column in timely fashion. ${ }^{15}$ To further assess the applicability of nitromethane as a selective quenching agent for HPLC analysis we report the effect that nitromethane has on the fluorescence emission behavior of 96 different alternant and nonalternant PAHs dissolved in a binary ethyl acetate/acetonitrile solvent mixture.

\section{MATERIALS AND METHODS}

Synthetic references and/or commercial suppliers for the PAH solutes contained in Table I are listed in our earlier papers. Stock solutions were prepared by dissolving the solutes in dichloromethane. Small aliquots of the stock solutions were transferred into test tubes, allowed to evaporate, and diluted with the solvent of interest. Final solute concentrations were sufficiently dilute to minimize inner-filtering artifacts. Solvents were of HPLC, spectroquality, or AR grade, purchased commercially from either Aldrich or Fisher Scientific, and the resulting solutions were optically dilute (absorbances $\left.\mathrm{cm}^{-1}<0.01\right)$ at all wavelengths, except for the nitromethane quenching studies.

Absorption spectra were recorded on a Milton Roy Spectronic 1001 Plus and a Hewlett-Packard 8450A photodiode array spectrophotometer in the usual manner. The fluorescence spectra were run on a Shimadzu RF$5000 \mathrm{U}$ spectrofluorometer with the detector set at high sensitivity. Solutions were excited at the wavelengths listed in Table I. Fluorescence data were accumulated in a $1-\mathrm{cm}^{2}$ quartz cuvette at $19^{\circ} \mathrm{C}$, ambient room temperature, with excitation and emission slit width settings of $15 \mathrm{~nm}$ and $3 \mathrm{~nm}$, respectively. For solvent polarity determinations involving pyrene, the emission slit width was closed to $1.5 \mathrm{~nm}$ so that the calculated intensity ratios could be compared directly with literature values. ${ }^{24}$ Street and $A c r e e^{25}$ had previously shown that emission intensity ratios vary with emission slit setting.

Emission intensities associated with the quenching study were corrected for primary inner-filtering artifacts arising from the absorption of excitation radiation. Many of the PAHs have excitation wavelengths in the 300-320 $\mathrm{nm}$ spectral region, and a few drops of nitromethane gave solutions having appreciable absorbances. Mathematical expressions, computational procedures, and interrogation zone dimensions are given elsewhere. ${ }^{17-19,26,27}$ Every effort was made to work at solution absorbances below $A \mathrm{~cm}^{-1} \leq 0.95\left(f_{\text {prim }} \leq 3.00\right)$ where the inner-filtering correction equation is valid. Secondary inner-filtering corrections were not necessary in the present study since nitromethane is "optically transparent" in most of these PAHs' emission ranges.

\section{RESULTS AND DISCUSSION}

Previous studies involving nitromethane as a selective quenching agent for discriminating between alternant vs. nonalternant PAHs utilized either neat acetonitrile or a binary aqueous/acetonitrile mixture (20:80\% by volume). Such solvents work well as mobile phases in HPLC separations for those PAHs that contain six rings or less, but different solvent strengths must be employed for larger PAHs having 6-10 rings. Toluene/acetonitrile or ethyl acetate/acetonitrile mixtures have served well in the past as HPLC mobile-phase solvents for the separation of the larger PAHs. ${ }^{15,28}$ Table I compares the experimental results regarding the ability of nitromethane to act as a selective quenching agent for alternant vs. nonalternant PAHs in the aqueous/acetonitrile mixture and toluene/acetonitrile mixture (40:60 percent by volume) utilized earlier, ${ }^{30}$ and an ethyl acetate/acetonitrile mixture (40:60 percent by volume). While a published paper ${ }^{16}$ has alluded to the fact that solvent polarity affects nitromethane's ability to act as a selective quenching agent, there was no in-depth study ever reported until we recently compared the aqueous/acetonitrile and toluene/acetonitrile mixtures. ${ }^{30}$

Examination of Table I reveals that the toluene/acetonitrile mixture $(\mathrm{Py}=1.49)$ and ethyl acetate/acetonitrile $(\mathrm{Py}=1.68)$, although less polar than the aqueous/ acetonitrile mixture (observed $\mathrm{Py}=1.60^{29} ; \mathrm{Py}=1.80$ after correction for slit width effects ${ }^{25}$ ) or pure acetonitrile $(\mathrm{Py}=1.79),{ }^{24}$ can effectively be used as an HPLC solvent when nitromethane is used to selectively quench the fluorescence emission signals of alternant vs. nonalternant PAHs. Of the 96 compounds examined, only eleven behave differently in the ethyl acetate/acetonitrile solvent mixtures as compared to either the aqueous/acetonitrile solvent mixture or neat acetonitrile. As in the toluene/acetonitrile solvent study, seven of the exceptions noted were coronene derivatives. Our initial studies utilized the aqueous/acetonitrile mixtures, but after a more exhaustive investigation involving 96 compounds we found that, except for the aforementioned coronene derivatives, the selective quenching rule is obeyed in neat acetonitrile as well. Since then, we have employed acetonitrile, rather than the aqueous/acetonitrile mixture, to enhance the solubility of the larger PAHs.

Two nonalternant compounds, naphtho[2,3b]fluoranthene and benzo[k]fluoranthene, that were exceptions to the nitromethane selective quenching rule in both aqueous/acetonitrile and toluene/acetonitrile mixtures are no longer exceptions when dissolved in the ethyl acetate/acetonitrile solvent. Interestingly, the two benzo[k]fluoranthene derivatives, 3-methoxybenzo[k]fluoranthene and 3-hydroxybenzo[k]fluoranthene, are quenched in ethyl acetate/acetonitrile even though the parent compound was not quenched. In both toluene/ acetonitrile and ethyl acetate/acetonitrile solvents, benzo[rst]dinaphtho[2,1,8,7defg; $\left.2^{\prime}, 1^{\prime}, 8^{\prime}, 7^{\prime} \mathrm{ijkl}\right]$ pentaphene (BNP) was not quenched by nitromethane. BNP should have been quenched on the basis of the fact that it is an alternant compound. Other than the coro- 
TABLE 1. Summary of excitation wavelength, probe character, and fluorescence quenching (by nitromethane) data for alternant and nonalternant PAHs.

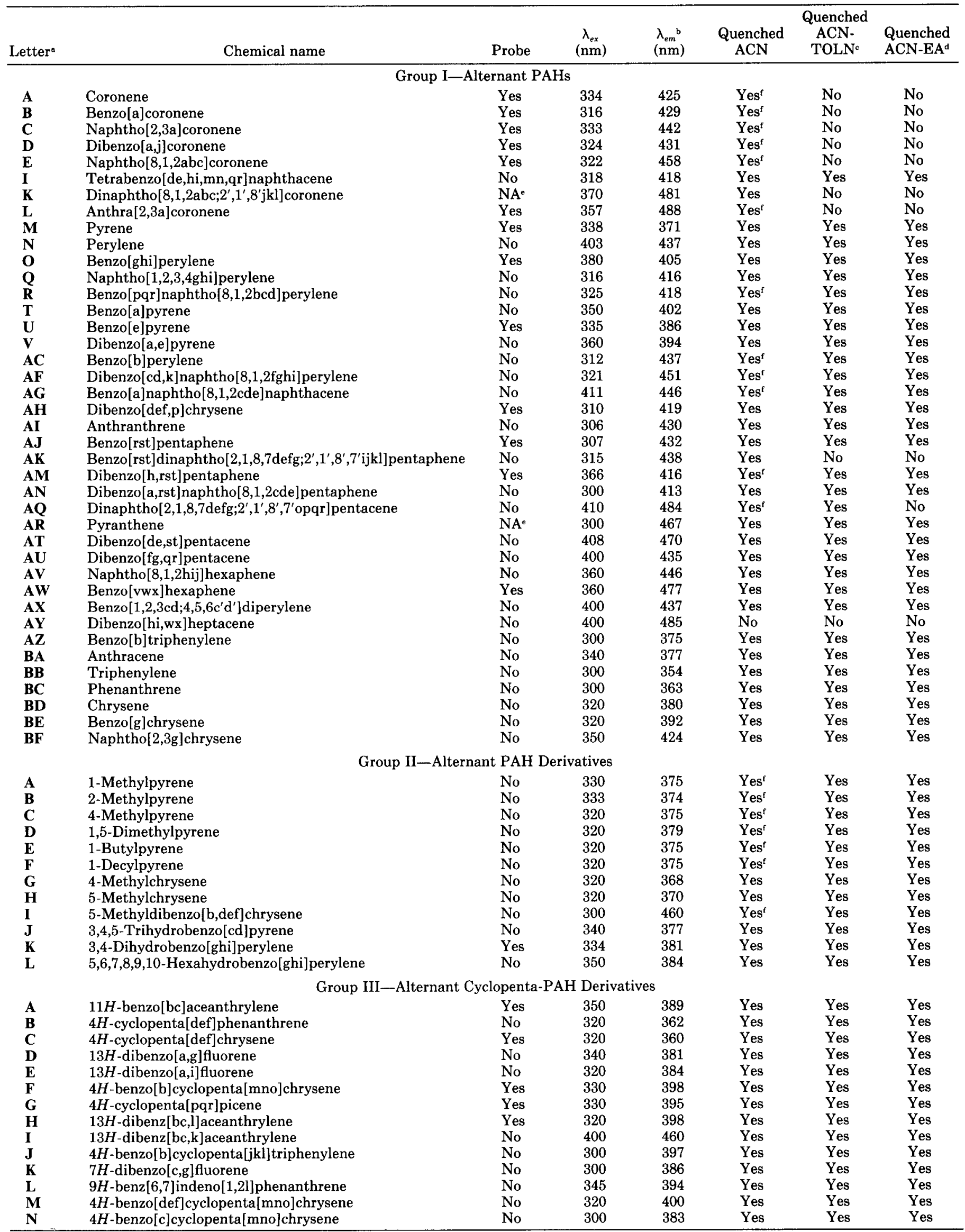




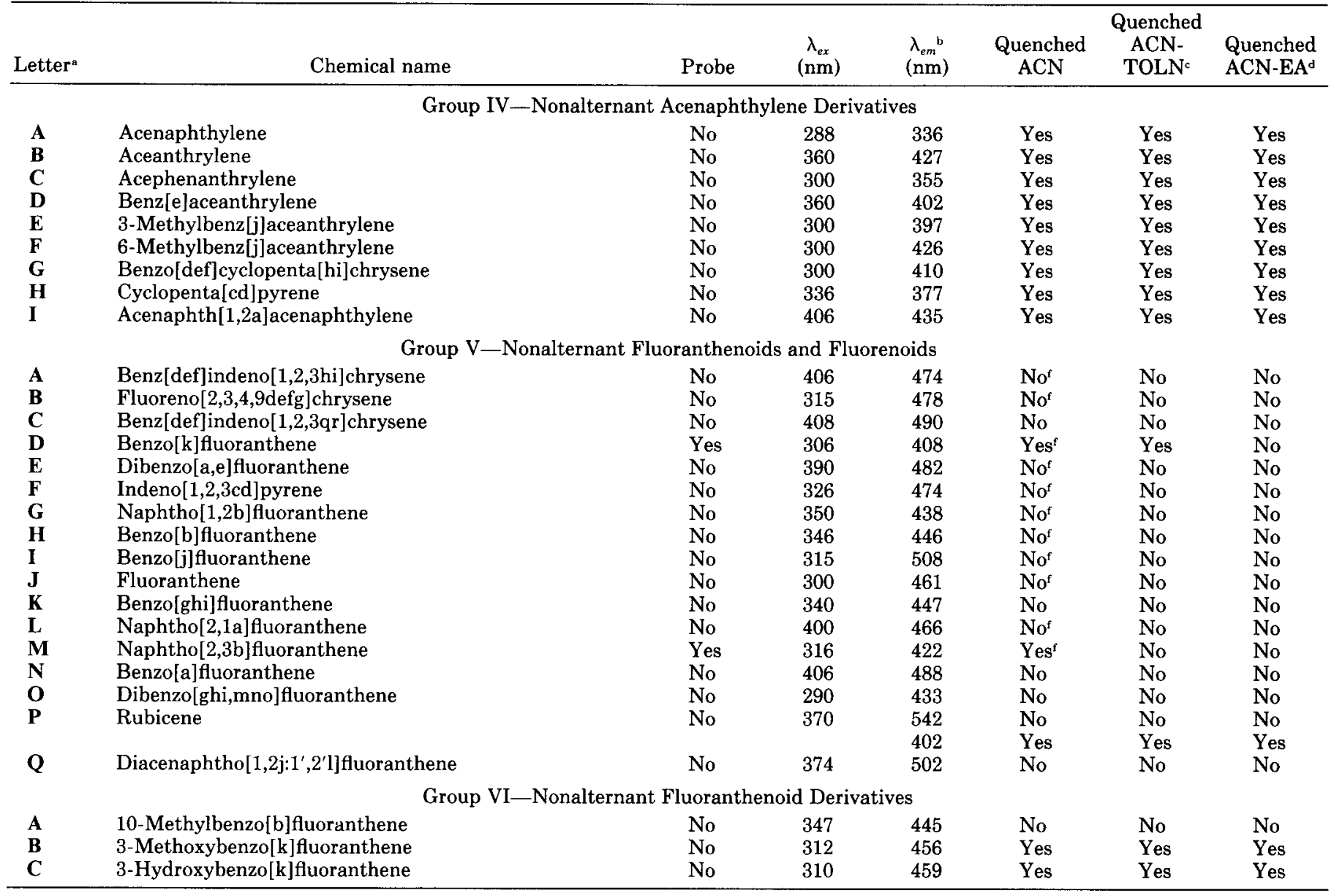

Molecular structures are given in Refs. 17-19, 23, 26, 30, and 31.

' Emission wavelength used in the nitromethane quenching studies.

c Quenching studies performed in a binary toluene/acetonitrile solvent mixture (40:60 percent by volume).

d Quenching studies performed in a binary ethyl acetate/acetonitrile solvent mixture (40:60 percent by volume).

e NA indicates that the PAC is unacceptable as a solvent polarity probe molecule because a common set of bands could not be assigned in all solvents studied. The molecule may exhibit selective enhancement of one (or more) fluorescence emission band(s).

${ }^{\mathrm{f}}$ Quenching studies performed in a binary aqueous/acetonitrile solvent mixture (20:80 percent by volume). Quenching of coronene and benzo[a]coronene fluorescence emission, after correction for primary inner-filtering and dilution, amounted to about a $20 \%$ reduction in signal intensity. Much larger reductions were observed for many of the other alternant PAHs studied.

nene derivatives, BNP and dinaphtho[2,1,8,7defg; $2^{\prime}, 1^{\prime}, 8^{\prime}, 7^{\prime}$ opqr]pentacene remain the only other major exceptions in the ethyl acetate/acetonitrile binary solvent mixture when compared to the more polar aqueous/acetonitrile system. Results of the present investigation clearly indicate that a solute's quenching behavior can be directly affected by the polarity of the surrounding solvent.

\section{ACKNOWLEDGMENTS}

This work is supported in part by grants from the National Science Foundation (Grant No. CTS-8922485), by the University of North Texas Research Council, and by a National Science Foundation Doctoral Research Fellowship awarded to S. A. Tucker.

1. J. Rein, C. M. Cork, and K. G. Furton, J. Chromatogr. 545, 149 (1991).

2. B. W. Wright, S. R. Frye, D. G. McMinn, and R. D. Smith, Anal. Chem. 59, 640 (1987)

3. S. B. Hawthorne and D. J. Miller, J. Chromatogr. 403, 63 (1987).

4. S. B. Hawthorne, J. J. Langenfeld, D. J. Miller, and M. D. Burford, Anal. Chem. 64, 1614 (1992).
5. B. W. Wright, C. W. Wright, R. W. Gale, and R. D. Smith, Anal. Chem. 59, 38 (1987).

6. P. J. A. Fowlie and T. L. Bulman, Anal. Chem. 58, 721 (1986).

7. G. A. Junk and J. J. Richard, Anal. Chem. 58, 962 (1986).

8. S. A. Wise, L. C. Sander, R. Lapouyande, and P. Garrigues, J. Chromatogr. 514, 111 (1990).

9. L. C. Sander and S. A. Wise, Anal. Chem. 59, 2309 (1987).

10. L. C. Sander and S. A. Wise, J. High Resolut. Chromatogr. Chromatogr. Commun. 11, 383 (1988).

11. L. C. Sander and S. A. Wise, Anal. Chem. 61, 1749 (1989).

12. J. C. Fetzer, in Chemical Analysis of Polycyclic Aromatic Compounds, T. Vo-Dinh, Ed. (John Wiley and Sons, New York, 1989), Chap. 3.

13. L. C. Sander, R. M. Parris, S. A. Wise, and P. Garrigues, Anal. Chem. 63, 2589 (1991).

14. J. C. Fetzer and W. R. Biggs, J. Chromatogr. 386, 87 (1987).

15. J. C. Fetzer and W. R. Biggs, Chromatographia 27, 118 (1989).

16. G.-P. Blümer and M. Zander, Fresenius Z. Anal. Chem. 296, 409 (1979).

17. S. A. Tucker, W. E. Acree, Jr., B. P. Cho, R. G. Harvey, and J. C. Fetzer, Appl. Spectrosc. 45, 1699 (1991).

18. V. L. Amszi, Y. Cordero, B. Smith, S. A. Tucker, W. E. Acree, Jr., C. Yang, E. Abu-Shaqara, and R. G. Harvey, Appl. Spectrosc. 46, 1156 (1991).

19. S. A. Tucker, H. Darmodjo, W. E. Acree, Jr., J. C. Fetzer, and M. Zander, Appl. Spectrosc. 46, 1260 (1992). 
20. H. Dreeskamp, E. Koch, and M. Zander, Z. Naturforsch. 30A, 1311 (1975).

21. U. Breymann, H. Dreeskamp, E. Koch, and M. Zander, Chem. Phys. Lett. 59, 68 (1978).

22. S. H. Chen, C. E. Evans, and V. L. McGuffin, Anal. Chim. Acta 246, 65 (1991).

23. S. A. Tucker, H. C. Bates, V. L. Amszi, W. E. Acree, Jr., H. Lee, P. D. Raddo, R. G. Harvey, J. C. Fetzer, and G. Dyker, Anal. Chim. Acta 278, 269 (1993).

24. D. C. Dong and M. A. Winnik, Can. J. Chem. 62, 2560 (1984).

25. K. W. Street, Jr., and W. E. Acree, Jr., Analyst 111, 1197 (1986).

26. S. A. Tucker, W. E. Acree, Jr., J. C. Fetzer, and J. Jacob, Polycyclic Aromat. Compds. 3, 1 (1992).
27. S. A. Tucker, V. L. Amszi, and W. E. Acree, Jr., J. Chem. Educ. 69, A8 (1992).

28. P A. Peaden, M. L. Lee, Y. Hirata, and M. V. Novotny, Anal. Chem. 52, 2268 (1980).

29. K. W. Street, Jr., and W. E. Acree, Jr., J. Liq. Chromatogr. 9, 2799 (1986).

30. S. A. Tucker, W. E. Acree, Jr., J. C. Fetzer, R. G. Harvey, M. J. Tanga, P.C. Cheng, and L. T. Scott, Appl. Spectrosc. 47, 715 (1993).

31. W. E. Acree, Jr., S. A. Tucker, and J. C. Fetzer, Polycyclic Aromat. Compds. 1, 75 (1991). 